\title{
First mitotic spindle formation is led by sperm centrosome- dependent MTOCs in humans
}

\author{
Yoshiteru Kai®, Hiroomi Kawano and Naoki Yamashita \\ Reproductive Medicine Research Center, Yamashita Shonan Yume Clinic, Fujisawa, Kanagawa, Japan \\ Correspondence should be addressed to Y Kai; Email: y.kai@ysyc-yumeclinic.com
}

\begin{abstract}
Unlike in mice, multinucleated blastomeres appear at a high frequency in the two-cell-stage embryos in humans. In this Point of View article, we demonstrate that the first mitotic spindle formation led by sperm centrosome-dependent microtubule organizing centers may cause a high incidence of zygotic division errors using human tripronuclear zygotes.

Reproduction (2021) 161 V19-V22
\end{abstract}

After penetration of the oocyte by sperm, each parental haploid genome forms a pronucleus in the zygote. The haploid genomes of the oocyte and sperm fuse after the breakdown of pronuclear membrane, just before the first cleavage. It was believed that, at the time of fusion, a single bipolar microtubule system self-assembled around both the parental genomes in the zygote (Fitzharris 2009, Courtois et al. 2012, Coelho et al. 2013, Duncan et al. 2020). However, a recent study reported the formation of two bipolar spindles in the mouse zygote, followed by an independent congression of the maternal and paternal genomes (Reichmann et al. 2018). This mechanism likely contributes to the proper divisions that maintain the chromosome stability during the first cleavage. In mouse oocytes, spindle microtubules are nucleated by multiple acentriolar microtubule organizing centers (MTOCs) because of the absence of canonical centrosomes (Nakamura et al. 2001). In humans, the sperm centrosome functions as MTOC and is responsible for nucleating the spindle microtubules (Sathananthan et al. 1991, Kai et al. 2015). Thus, formation of the first mitotic spindle may differ between mice and humans. Therefore, the aim of this study was to reveal the process of first mitotic spindle formation in human fertilized oocytes focusing on the sperm centrosome.

To examine how the sperm centrosomes contribute to MTOC formation, we used human tripronuclear (3PN) zygotes derived from conventional in vitro fertilization (c-IVF) and intracytoplasmic sperm injection (ICSI). 3PN zygotes with two polar bodies derived from c-IVF possess four centrosomes (dispermic fertilization), whereas 3PN zygotes with a single polar body derived from ICSI possess two centrosomes (diginyc fertilization) (Fig. 1A, Kai et al. 2015). We took advantage of this difference to analyze the role of the sperm centrosome as the MTOC during the first cleavage.
Zygotes were fixed at consecutive stages of development and analyzed using immunofluorescence staining (Fig. 1B and C). 3PN zygotes derived from c-IVF showed four pericentrin dots (Fig. 1B), whereas 3PN zygotes derived from ICSI exhibited two pericentrin dots (Fig. 1C). In pro-metaphase, we observed an independent group of chromosomes derived from each pronucleus and MTOCs formed by the sperm centrosome at the core. Microtubules from each MTOC extended toward the chromosomes in early metaphase; a quadrupolar spindle (Fig. 1B) was formed in 3PN zygotes from c-IVF, and a bipolar spindle (Fig. 1C) was formed in 3PN zygotes from ICSI by MTOCs at the apex of the zygote after chromosome alignment. In pro-metaphase, microtubules were found to extend from MTOCs to the nearest chromosome (Fig. 1B and C). Microtubule assembly was observed on H3K9me3positive oocyte-derived chromosomes; therefore, we believe that, whether a chromosome is surrounded by microtubules depends on the location of the MTOCs, irrespective of the chromosomal origin (Fig. 1D and Video 1).

\section{Video 1}

3D movie of immunofluorescence staining of tripronuclear (3PN) zygotes derived from conventional in vitro fertilization (c-IVF) arrested at pro-metaphase. This video is available from the online version of the article at https://doi.org/10.1530/REP-21-0061.

Live-imaging analysis of 3PN zygotes derived from c-IVF revealed that four MTOCs appeared around the three pronuclei just before the disappearance of the pronuclear membrane (Fig. 1E and Video 2), and microtubules then extended from MTOCs toward the 
A

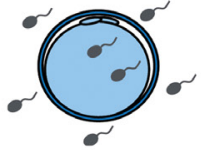

Dispermic fertilization in c-IVF

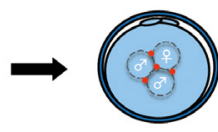

3PN zygote containing

four centrosomes

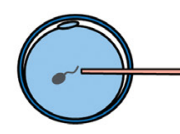

Failure of second polar body
extrusion in ICSI

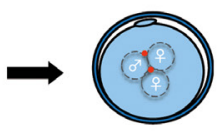

3PN zygote containing

B

a-tubulin Pericentrin Chromosome Merge

C

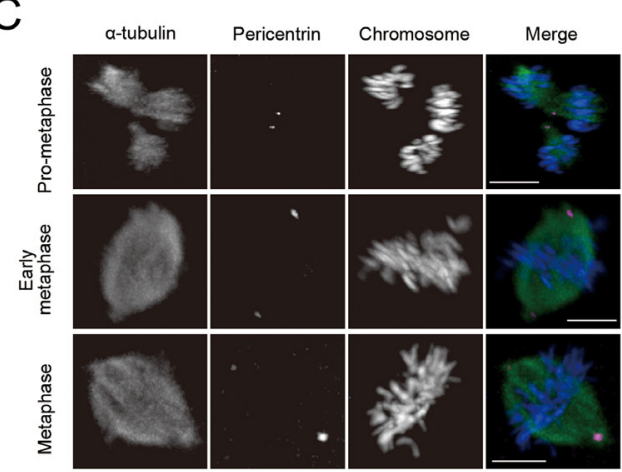

D
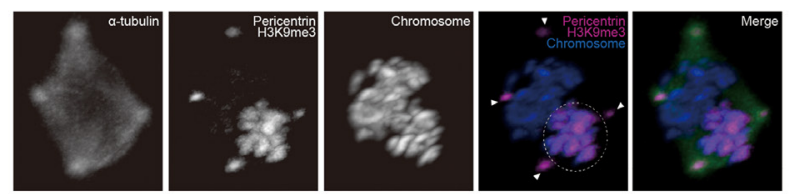

E

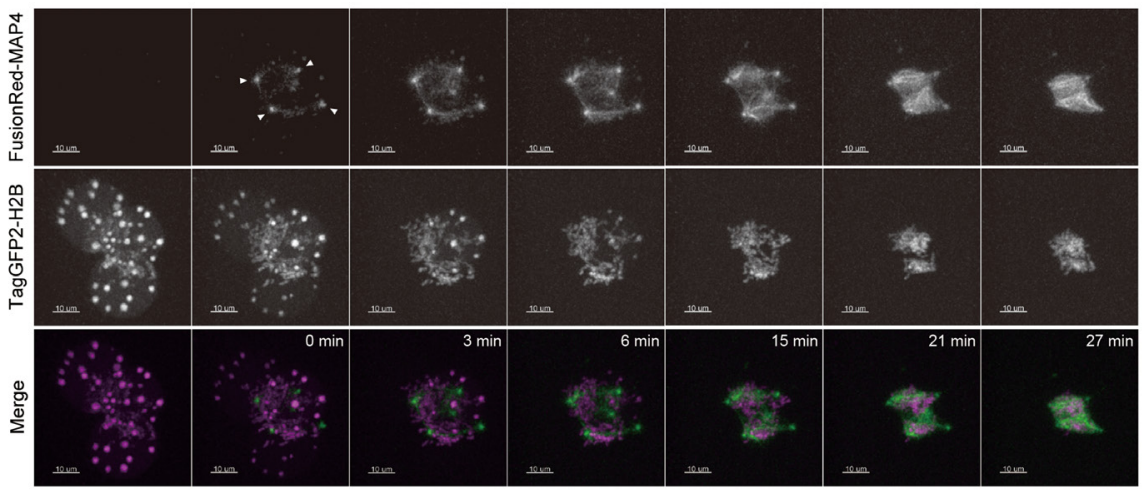

F
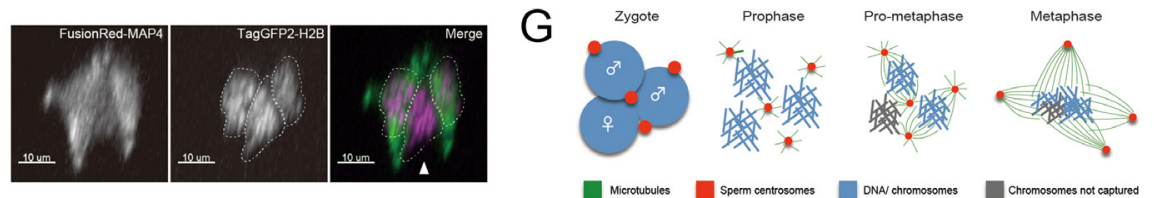

Figure 1 First mitotic spindle formation dependent on the sperm centrosome. (A) Origins of the 3PN zygotes. (B) Immunofluorescence staining of tripronuclear (3PN) zygotes derived from conventional in vitro fertilization (c-IVF) and fixed at consecutive stages of development (80x magnification). (C) Immunofluorescence staining of 3PN zygotes derived from intracytoplasmic sperm injection (ICSI) and fixed at consecutive stages of development (80x magnification). (D) Immunofluorescence staining of 3PN zygotes derived from c-IVF fixed at prometaphase (88x magnification). White arrowheads indicate the four MTOCs originating from sperm centrosomes, whereas the dotted line indicates the chromosomes derived from the oocyte. Maximum z projections of confocal sections of zygotes at pro-metaphase, early metaphase, and metaphase are also shown. Microtubules were stained with antibodies against $\alpha$-tubulin (green); MTOCs, antibodies against pericentrin (magenta) and chromosome, NucBlue I stain (blue). Scale bar, $10 \mu \mathrm{m}$. (E) Time-lapse imaging of a tripronuclear (3PN) zygote derived from conventional in vitro fertilization (c-IVF) and expressing TagGFP2-H2B (magenta: chromosome) and FusionRed-MAP4 (green: microtubules, $40 \times$ magnification). ' $0 \mathrm{~min}^{\prime}$ indicates the start of pronuclear disappearance. White arrowheads indicate four MTOCs that originated from sperm centrosomes. Scale bar, $10 \mu \mathrm{m}$. (F) At $21 \mathrm{~min}$ after disappearance of the pronuclear membrane, images of Fig. 1E were rotated to highlight the positional relationship between the three groups of chromosomes surrounded by dotted line and microtubules (60× magnification).

Chromosomes derived from one of the three pronuclei are not assembled with microtubules (white arrowhead). Scale bar, $10 \mu \mathrm{m}$. . (G) A proposed schematic diagram of the formation of the first mitotic spindle in human 3PN zygotes derived from c-IVF. Four sperm centrosomedependent MTOCs are formed at prophase and microtubules preferentially extend toward the chromosomes closer to MTOCs at pro-metaphase. At this phase, failure of chromosome capture by microtubules occurs. Next, a single quadrupolar first mitotic spindle is formed as the chromosomes move up the equatorial plane at metaphase. 
chromosomes, beginning to form a mitotic spindle as the chromosomes moved to the center of the oocyte. Interestingly, one of the three groups of assembled chromosomes showed no microtubule assembly in prometaphase (Fig. 1F and Video 3). Similar results were obtained in all six $3 \mathrm{PN}$ zygotes subjected to live-imaging analysis.

\section{Video 2}

Time-lapse movie of a tripronuclear (3PN) zygote derived from conventional in vitro fertilization (c-IVF) that expresses the chromosome marker TagGFP2-H2B (green) and microtubule marker FusionRed-MAP4 (red). This video is available from the online version of the article at https://doi.org/10.1530/REP-21-0061.

\section{Video 3}

3D movie of live-imaging analysis at $21 \mathrm{~min}$ after the disappearance of the pronuclear membrane of tripronuclear (3PN) zygotes derived from the conventional in vitro fertilization (c-IVF) and showing the positional relationship of the chromosomes and microtubules. This video is available from the online version of the article at https://doi.org/10.1530/ REP-21-0061.

Our results indicate that the fertilized zygote in humans form the first mitotic spindle from the sperm centrosome, unlike in mice (Fig. 1G). In mice, multiple MTOCs appear around each pronucleus after the disappearance of the pronuclear membrane, and they contribute to the formation of dual-spindle around each parental chromosome (Reichmann et al. 2018). If this process in humans was similar to that in mice, the $3 \mathrm{PN}$ zygote should have formed three spindles; however, this was not observed. This may be because of the difference in the MTOCs formation in the oocytes of mice and humans. Our results revealed that microtubules are nucleated in sperm centrosomes, which form MTOCs after the disappearance of the pronuclear membrane and begin to extend toward the chromosomes. Next, a spindle with the sperm centrosome-dependent MTOC as the pole is formed as the chromosomes move to the equatorial plane (Fig. 1G). Notably, the microtubules preferentially extended to chromosomes that were closer to the MTOCs, regardless of their male or female origin. In other words, chromosomes farther away from the MTOCs are unlikely to be surrounded by microtubules. In mouse, the chromosome-led pathway of microtubule assembly is predominant, while, in human embryos, the centrosome-led 'search and capture' pathway is dominant. Because, normal fertilized oocytes in humans have two sperm-derived centrosomes (Kai et al. 2015), two sperm centrosome-dependent MTOCs should form during the first cleavage. However, two
MTOCs may not be sufficient to completely enclose the physically separated female and male chromosomes with microtubules, thus explaining the high frequency of zygotic division errors that contribute to the unstable nature of human chromosomes (Aguilar et al. 2016).

Our findings suggest that it is necessary to consider the differences in the mechanism of MTOC organization in fertilized mouse oocytes when studying human fertilization. Improving the understanding of the chromosome separation machinery in early human embryos may contribute to the development of new assistive reproductive techniques.

\section{Declaration of interest}

The authors declare that there is no conflict of interest that could be perceived as prejudicing the impartiality of this point of view.

\section{Funding}

This research did not receive any specific grant from any funding agency in the public, commercial or not-for-profit sector.

\section{Author contribution statement}

Y K designed the study, performed all experiments, and wrote the manuscript. $\mathrm{H} \mathrm{K}$ collected the tripronuclear zygotes. $\mathrm{N}$ $Y$ supervised the study and obtained informed consent from patients.

\section{Acknowledgements}

The authors thank Takeshi Matsui (RIKEN Center for Integrative Medical Science) for his assistance with plasmid vector construction and Takashi Hiiragi (European Molecular Biology Laboratory) for his helpful comments on the manuscript. The authors are grateful to the staff of the Yamashita Shonan Yume Clinic for their assistance and encouragement.

\section{References}

Aguilar J, Rubio I, Muñoz E, Pellicer A \& Meseguer M 2016 Study of nucleation status in the second cell cycle of human embryo and its impact on implantation rate. Fertility and Sterility 106 291.e2-299.e2. (https://doi.org/10.1016/j.fertnstert.2016.03.036)

Albertini DF, Herman B \& Sherline P 1984 In vivo and in vitro studies on the role of HMW-MAPs in Taxol-induced microtubule bundling. European Journal of Cell Biology 33 134-143.

Coelho PA, Bury L, Sharif B, Riparbelli MG, Fu J, Callaini G, Glover DM \& Zernicka-Goetz M 2013 Spindle formation in the mouse embryo requires Plk4 in the absence of centrioles. Developmental Cell 27 586-597. (https://doi.org/10.1016/j.devcel.2013.09.029)

Courtois A, Schuh M, Ellenberg J \& Hiiragi T 2012 The transition from meiotic to mitotic spindle assembly is gradual during early mammalian development. Journal of Cell Biology 198 357-370. (https://doi. org/10.1083/jcb.201202135) 
Duncan FE, Schindler K, Schultz RM, Blengini CS, Stein P, Stricker SA, Wessel GM \& Williams CJ 2020 Unscrambling the oocyte and the egg: clarifying terminology of the female gamete in mammals. Molecular Human Reproduction 26 797-800. (https://doi.org/10.1093/molehr/ gaaa066)

Fitzharris G 2009 A shift from kinesin 5-dependent metaphase spindle function during preimplantation development in mouse. Development 136 2111-2119. (https://doi.org/10.1242/dev.035089)

Kai Y, Iwata K, Iba Y \& Mio Y 2015 Diagnosis of abnormal human fertilization status based on pronuclear origin and/or centrosome number. Journal of Assisted Reproduction and Genetics 32 1589-1595. (https://doi.org/10.1007/s10815-015-0568-1)

Nakamura S, Terada Y, Horiuchi T, Emuta C, Murakami T, Yaegashi N \& Okamura K 2001 Human sperm aster formation and pronuclear decondensation in bovine eggs following intracytoplasmic sperm injection using a piezo-driven pipette: a novel assay for human sperm centrosomal function. Biology of Reproduction 65 1359-1363. (https:// doi.org/10.1095/biolreprod65.5.1359)
Reichmann J, Nijmeijer B, Hossain MJ, Eguren M, Schneider I, Politi AZ, Roberti MJ, Hufnagel L, Hiiragi T \& Ellenberg J 2018 Dual-spindle formation in zygotes keeps parental genomes apart in early mammalian embryos. Science 361 189-193. (https://doi.org/10.1126/science. aar7462)

Sathananthan AH, Kola I, Osborne J, Trounson A, Ng SC, Bongso A \& Ratnam SS 1991 Centrioles in the beginning of human development. PNAS 88 4806-4810. (https://doi.org/10.1073/pnas.88.11.4806)

Received 13 February 2021

First decision 25 February 2021

Revised Manuscript received 9 March 2021

Accepted 19 March 2021 